\title{
Comparative Study between Mesh Fixation vs. Non-Fixation in Totally Extraperitoneal Hernioplasty for Inguinal Hernia
}

\section{Osama Hasan Abd-Raboh, Mohamed Abdallah Hablus, Ahmed Abdelfatah Elshora, Sherif Abdelfatah Saber}

General Surgery Department, Tanta University Hospitals, Tanta, Egypt

Email address:

dr_osama_hassan@med.tanta.edu.eg(O.H. Abd-Raboh)

\section{To cite this article:}

Osama Hasan Abd-Raboh, Mohamed Abdallah Hablus, Ahmed Abdelfatah Elshora, Sherif Abdelfatah Saber. Mesh Fixation VS. Non-

Fixation in Totally Extraperitoneal Hernioplasty for Inguinal Hernia, Journal of Surgery. Vol. 6, No. 1, 2018, pp. 23-28.

doi: $10.11648 /$ j.js.20180601.15

Received: January 13, 2018; Accepted: January 26, 2018; Published: February 19, 2018

\begin{abstract}
Aim of the work: Randomized comparative study comparing outcome of mesh fixation versus non-fixation in TEP technique for inguinal hernia repair. Methods: In Tanta University Hospitals, over the period from February 2015 to October 2017, 58 patients with with 67 inguinal hernias (9 bilateral) undergoing TEP technique were randomized into 2 groups: Group A; mesh fixation 31 patients with 35 hernias. Group B; mesh non-fixation 27 patients with 32 hernias. Follow up was 6-17 months. Results: There was no statistical difference between 2 groups regarding operative time, postoperative complications, length of hospital stay and risk of chronic groin pain. Postoperative pain score was higher in Group A (mesh fixation). There was one case of hernia recurrence in group A (hernia and recurrence were both indirect), however, risk of hernia recurrence was not statistically significant. Conclusion: Mesh fixation and non-fixation in TEP are comparable regarding operative time, complications and hospital stay, while pain score was higher with mesh fixation. Mesh fixation does not increase the risk of chronic groin pain, while non-fixation does not increase hernia recurrence rate. Both techniques were proved safe.
\end{abstract}

Keywords: Inguinal Hernia, TEP, Totally Extraperitoneal, Mesh, Fixation, Tacker, Mesh Migration, Hernia Recurrence

\section{Introduction}

Although more difficult to master, there are several advantages of the TEP repair as compared to open techniques of inguinal hernioplasty. TEP has less postoperative pain with shorter convalescence, fewer hematomas, and deep space infections while the recurrence rates remain nearly equal to open techniques [1]. Some studies comparing different types of meshes and fixation methods revealed that non-fixation of mesh had higher rate of mesh migration [2]. Others said that mesh fixation does not add strength to the repair and its purpose is to prevent mesh displacement during first $24-48$ hours $[3,4]$. Despite the long period since Totally Extraperitoneal (TEP) approach was first described by McKernan and Laws in 1993, there is still debate about the clinical significance of mesh fixation.

\section{Aim of the Work}

This study was conducted to reveal any clinical or statistical difference between mesh fixation and non-fixation during TEP approach

\section{Patients and Methods}

This prospective study was conducted at General Surgery Department, Tanta University Hospitals, Egypt during the period from February 2015 to October 2017 (including follow up period of the study patients). 58 patients were randomized into 2 groups: Group A included 31 patients (4 bilateral cases giving total of 35 hernias) in which TEP was done with mesh fixation, while Group B included 27 patients ( 5 bilateral cases giving total of 32 hernias) in which TEP was done without mesh fixation. 


\subsection{Inclusion Criteria}

Adult patients that were aged above 18 years old were included in this study after informed consent. Patients with unilateral and bilateral inguinal hernias were candidates for this study.

\subsection{Exclusion Criteria}

Patients with huge scrotal, complicated or recurrent inguinal hernias were excluded from this study. Also, previous lower abdominal incision, pelvic operation and bad general condition of the patient, all were contraindications for inclusion in this study.

\subsection{Technique}

Under general anesthesia, we have done TEP technique with direct telescopic dissection of the midline preperitoneal tunnel for all patients. Technique details are described elsewhere [5]. Polypropylene mesh that measures $12 * 15 \mathrm{~cm}$ was used in every case to cover all potential inguinal defects. Mesh fixation was done in all cases of Group A at least in three points; pubic bone, upper medial and upper lateral corners taking care that no fixation was done in both triangles of pain and doom. Fixation method was either permanent non absorbable tacks (Figure 1, 2) or absorbable tacks (Figure 3). While in group B, the mesh was inserted without any fixation in all cases and desufflation was done under vision (Figure 4, 5 , 6). Drain was inserted in selected cases especially if there was excessive dissection of hernial sac from cord structures.

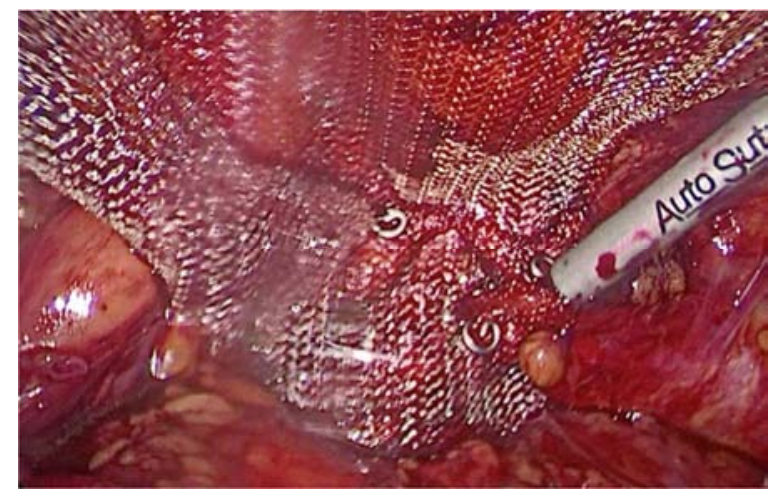

Figure 1. Group A; fixation with non-absorbable tacks.

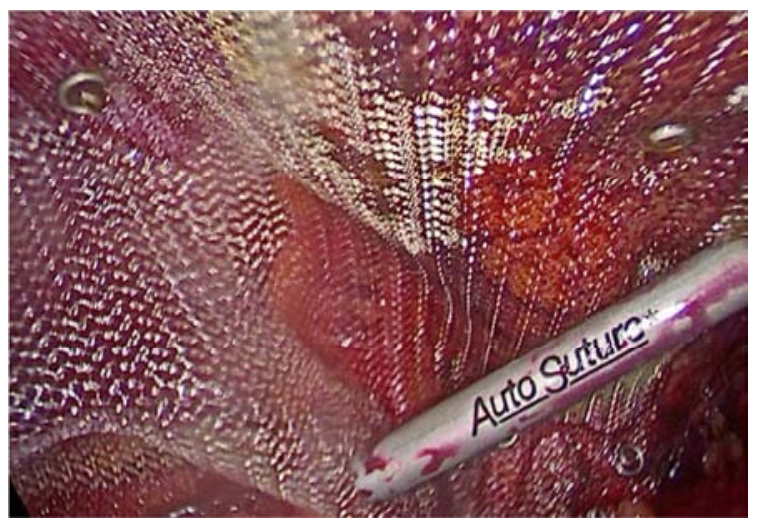

Figure 2. Group A; fixation with non-absorbable tacks.

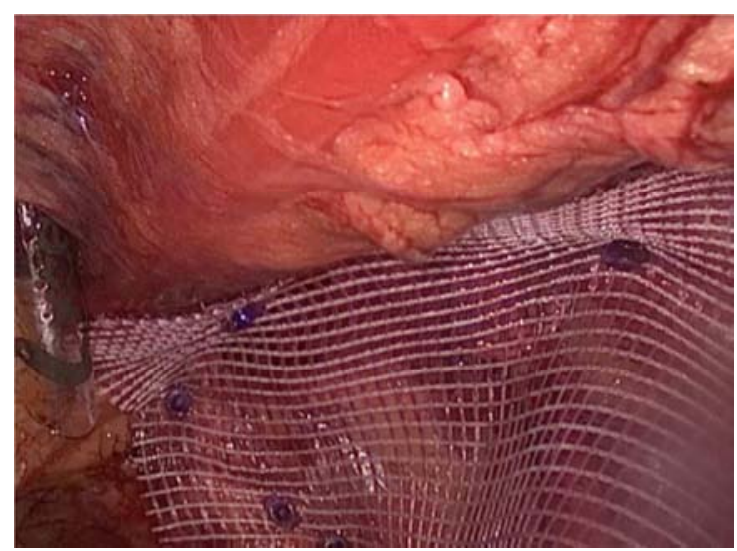

Figure 3. Group A; fixation with absorbable tacks.

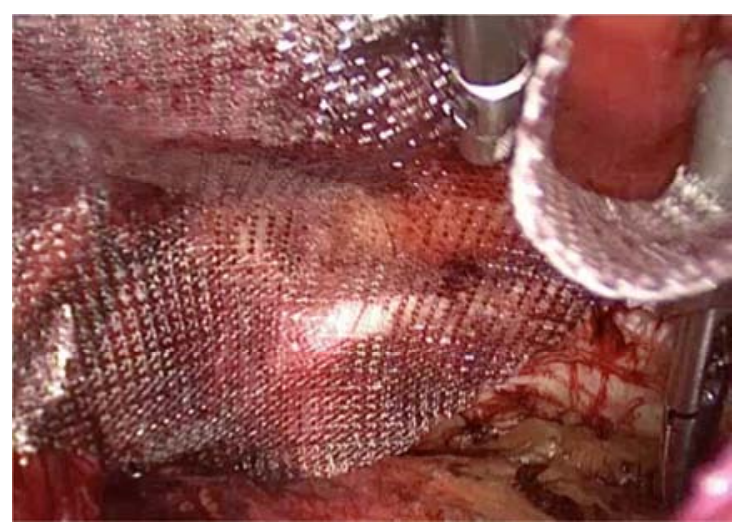

Figure 4. Group B; mesh non-fixation.

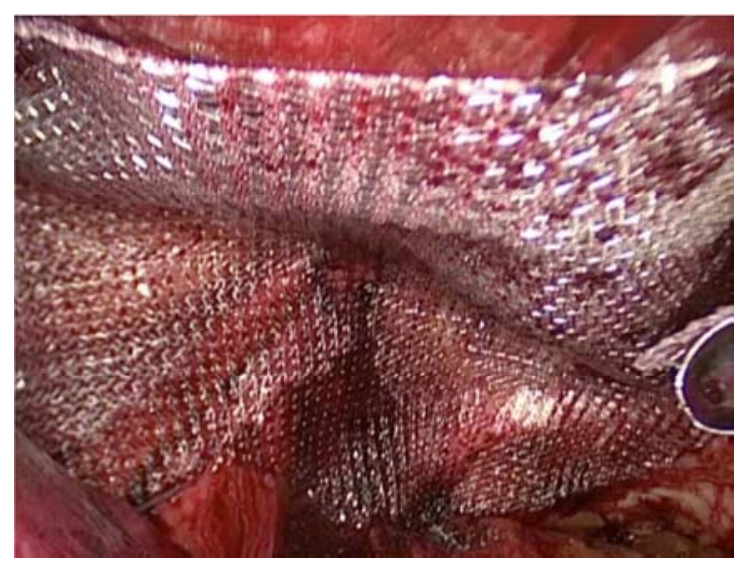

Figure 5. Group B; mesh non-fixation.

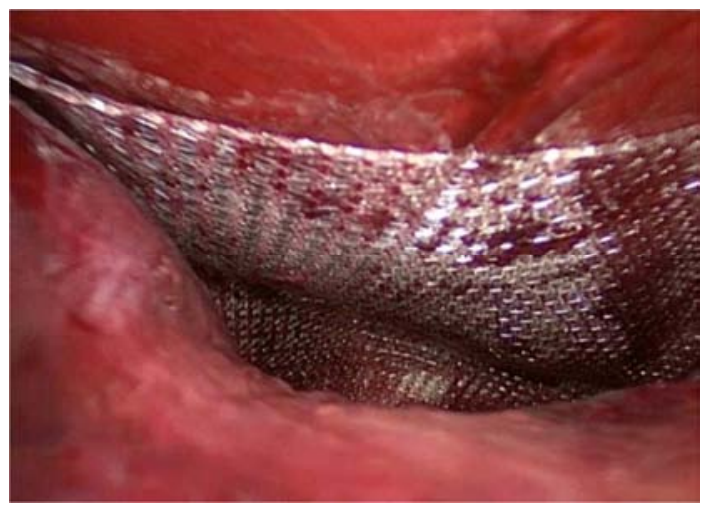

Figure 6. Group B; mesh non-fixation; desufflation under vision. 


\subsection{Postoperative Care}

All patients were hospitalized for at least the first 24 hours. Pain was assessed 24 hours post operative and patients were discharged only after they were fully mobile, with normal bowel habits and pain was controlled with oral analgesia. Any complication was recorded and managed. Patients were followed up for at least 6 months postoperative.

\section{Results}

This study included 58 patients ( 9 bilateral cases giving 67 total number of hernia repairs) randomized into 2 groups. Group A (mesh fixation group) included 31 patients with inguinal hernia; 27 unilateral hernias (16 right and 11 left sided) and 4 bilateral hernias giving total of 35 hernias. Group B (mesh non-fixation group) included 27 patients with inguinal hernia; 22 unilateral hernias (10 right and 12 left sided) and 5 bilateral hernias giving total of 32 hernias.

Patients' age ranged in group A from 19 - 62 years old and in group B 20 - 61 years (mean age was 35.84 and 36.7 years; P-value 0.787). One 39 years old female case in this study was in group A with left indirect inguinal hernia. Most of hernias were indirect inguinal hernias in both groups; 3 direct and 1 pantaloon hernias in group A (out of 35 hernias), while in group B there were 4 direct and 2 pantaloon hernias (out of 32 hernias) (P-value 0.676 ).

Operative time ranged from 45 to 105 minutes in group A and from 50 to 100 minutes in group B (mean operative time 67.43 and 66.41 minutes; P-value 0.765). In bilateral cases, operative time was measured for each side alone by adding the initial access time to both sides. Drain was inserted in 2 cases of group A and 3 cases of group B (P-value 0.528). Peritoneal tears occurred in 6 cases in group A (17.14\%) and in 5 cases in group B $(15.62 \%)$, however, these were small tears that didn't interfere with completion of the operation. There was no conversion from TEP either to TAPP or to open approach in any case of group A or B.

Pain on Visual Analogue Pain Scale at 24 hours postoperative was higher in Group A than group B (mean 3.48 and 2.89; P-value 0.046) (Table 1). Hospital stay ranged from 1 to 3 days in both groups (mean 1.26 and 1.22; P-value $0.803)$. Postoperative pain and hospital stay was significantly higher in bilateral than unilateral cases in group A (P-value 0.025 and 0.004 for pain and hospital stay), while this was not the case for group B (non-fixation) (P-value 0.057 for pain and 0.063 for hospital stay) (Table 2). Also, 24 postoperative pain was progressively increased with increasing operative time in both group A and B (P-value 0.001).

Table 1. Pain score is the only significant difference between Group A and B.

\begin{tabular}{|c|c|c|c|c|c|c|c|c|c|}
\hline & & Range & & & Mean & \pm & S. D & t. test & p. value \\
\hline \multirow{2}{*}{ Age } & Group A & 19 & - & 62 & 35.84 & \pm & 12.67 & \multirow{2}{*}{0.074} & \multirow{2}{*}{0.787} \\
\hline & Group B & 20 & - & 61 & 36.70 & \pm & 11.35 & & \\
\hline \multirow{2}{*}{ Operative time } & Group A & 45 & - & 105 & 67.43 & \pm & 14.52 & \multirow{2}{*}{0.090} & \multirow{2}{*}{0.765} \\
\hline & Group B & 50 & - & 100 & 66.41 & \pm & 13.27 & & \\
\hline \multirow{2}{*}{ Pain score after $24 \mathrm{~h}}$. & Group A & 2 & - & 6 & 3.48 & \pm & 1.23 & \multirow{2}{*}{4.182} & \multirow{2}{*}{$0.046^{*}$} \\
\hline & Group B & 2 & - & 5 & 2.89 & \pm & 0.93 & & \\
\hline Hospital stay & Group A & 1 & - & 3 & 1.26 & \pm & 0.58 & 0.063 & 0.803 \\
\hline \multirow{2}{*}{ Follow up } & Group A & 6 & - & 15 & 9.68 & \pm & 2.81 & \multirow{2}{*}{0.343} & \multirow{2}{*}{0.560} \\
\hline & Group B & 6 & - & 17 & 10.15 & \pm & 3.31 & & \\
\hline
\end{tabular}

Table 2. Impact of bilateral hernia on pain score 24 hours postoperative and hospital stay.

\begin{tabular}{|c|c|c|c|c|c|c|c|c|c|c|}
\hline & & & & & & Mean & \pm & S. D & t. test & p. value \\
\hline \multirow{4}{*}{ Group A } & \multirow{2}{*}{ Pain score after $24 \mathrm{~h}}$. & Unilateral & 2 & - & 5 & 3.30 & \pm & 1.14 & \multirow{2}{*}{5.563} & \multirow{2}{*}{$0.025^{*}$} \\
\hline & & Bilateral & 3 & - & 6 & 4.75 & \pm & 1.26 & & \\
\hline & \multirow{2}{*}{ Hospital stay } & Unilateral & 1 & - & 3 & 1.15 & \pm & 0.46 & \multirow{2}{*}{9.897} & \multirow{2}{*}{$0.004 *$} \\
\hline & & Bilateral & 1 & - & 3 & 2.0 & \pm & 0.82 & & \\
\hline \multirow{3}{*}{ Group B } & \multirow{2}{*}{ Pain score after $24 \mathrm{~h}$. } & Unilateral & 2 & - & 5 & 2.73 & \pm & 0.94 & \multirow{2}{*}{3.965} & \multirow{2}{*}{0.057} \\
\hline & & Bilateral & 3 & - & 4 & 3.60 & \pm & 0.55 & & \\
\hline & Hospital stay & Unilateral & 1 & - & 3 & 1.14 & \pm & 0.47 & 3.781 & 0.063 \\
\hline
\end{tabular}

Table 3. Drain insertion and postoperative complications.

\begin{tabular}{|c|c|c|c|c|c|c|}
\hline & & & Group A & Group B & $X^{2}$ & P-value \\
\hline \multirow{4}{*}{ Drain } & \multirow{2}{*}{0} & $\mathrm{~N}$ & 29 & 24 & \multirow{4}{*}{0.398} & \multirow{4}{*}{0.528} \\
\hline & & $\%$ & $93.5 \%$ & $88.9 \%$ & & \\
\hline & \multirow{2}{*}{1} & $\mathrm{~N}$ & 2 & 3 & & \\
\hline & & $\%$ & $6.5 \%$ & $11.1 \%$ & & \\
\hline \multirow{3}{*}{ Seroma } & \multirow{2}{*}{0} & $\mathrm{~N}$ & 29 & 26 & \multirow{3}{*}{0.222} & \multirow{3}{*}{0.637} \\
\hline & & $\%$ & $93.5 \%$ & $96.3 \%$ & & \\
\hline & 1 & $\mathrm{~N}$ & 2 & 1 & & \\
\hline Surgical emphysema & 0 & $\mathrm{~N}$ & 30 & 25 & \multirow{2}{*}{0.514} & \multirow{2}{*}{0.473} \\
\hline Suigiedi empinysenta & 0 & $\%$ & $96.8 \%$ & $92.6 \%$ & & \\
\hline
\end{tabular}




\begin{tabular}{lllllll}
\hline & & & Group A & Group B & $\mathbf{X}^{2}$ & P-value \\
\hline & \multirow{2}{*}{ Recurrence } & $\mathrm{N}$ & 1 & 2 & & \\
& & $\%$ & $3.2 \%$ & $3.4 \%$ & & \\
& 0 & $\mathrm{~N}$ & 34 & $100.0 \%$ & 0.933 & 0.335 \\
& & $\%$ & $97.1 \%$ & 0 & & \\
\hline
\end{tabular}

Follow up period for group A ranged from 6 to 15 months (mean $9.68 \pm 2.81$ months), while in group B from 6 to 17 months (mean 10.15 \pm 3.31 months). Postoperative complications included; seroma formation in 2 cases of group $\mathrm{A}$ and 1 case of group B (P-value 0.637) and surgical emphysema in 1 case of group A and 2 cases of group B (Pvalue 0.473 ) (Table 3). One case of hernia recurrence occurred in early postoperative period in male patient 37 years old with right indirect inguinal hernia treated by TEP repair with mesh fixation (group A). This patient suffered from vigorous vomiting in early postoperative recovery. After discussion with the patient and taking consent, management was done 13 days postoperatively; laparoscopic examination was done first showing the recurrent hernial defect with large sac (Figure 7), then open anterior approach tension free hernia repair was done with excision of large recurrent sac. Preperitoneal mesh previously put by TEP approach was apparently displaced above the internal ring and the recurrent sac (Figure 8).

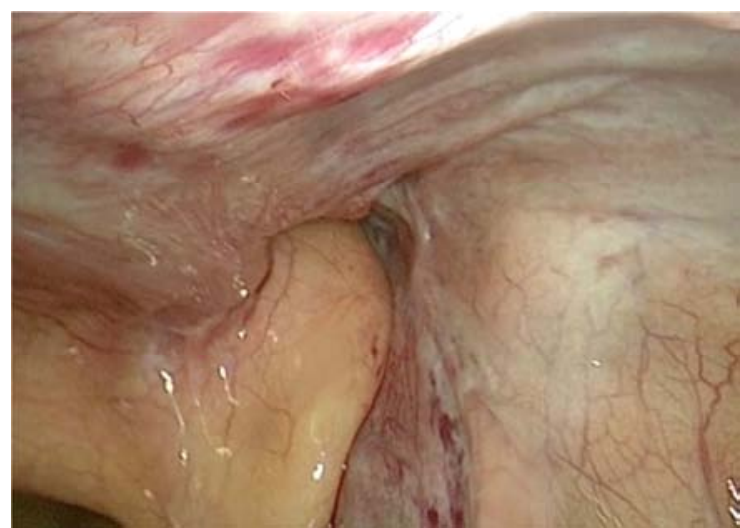

Figure 7. Recurrent right inguinal hernia, laparoscopic view.

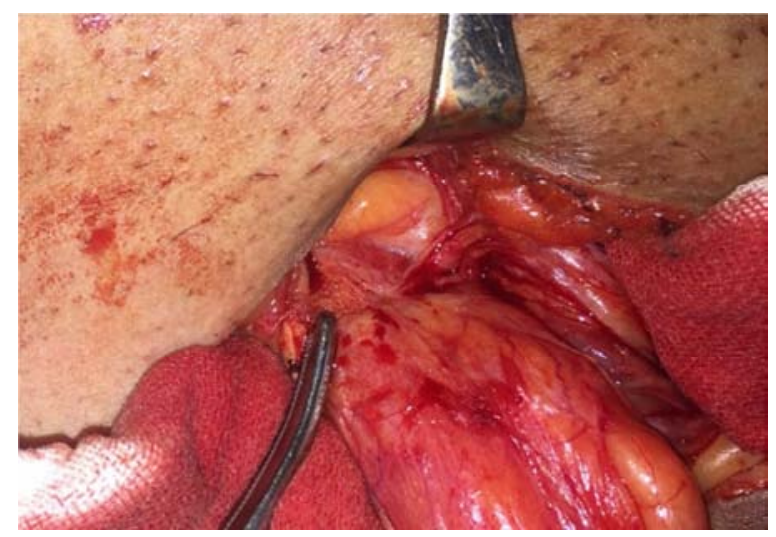

Figure 8. Recurrent right inguinal hernia (the same case); open anterior repair with the edge of the preperitoneal mesh elevated above the recurrent sac.

\section{Discussion}

\subsection{Demographic and Anatomical Data}

There was no significant variation between 2 groups regarding age and sex distribution. Also, numbers of bilateral cases, right or left, direct and indirect hernias did not show any remarkable difference.

\subsection{Operative Time and Hospital Stay}

Without any significant difference between 2 groups, mean operative time was comparable with other recent studies within the same study volume [6, 7, 8]. However, larger volume studies showed shorter operative time in both fixation and non-fixation of mesh [9].

Although some studies showed significantly shorter hospital stay in non-fixation group [8], in this study there was no difference between 2 groups. This may be due to study recommendation not to discharge patients before 24 pain score measurements.

\subsection{Complications}

No significant difference between 2 groups regarding postoperative complications. Minor complications included surgical emphysema and seroma formation that were managed conservatively in all patients. Being relatively small number of cases in this study, this study cannot identify any relation between the use of drain (2 and 3 cases in Group A and B) and causation of or prevention of seroma formation. A large meta-analysis of 6 studies also revealed no significant difference between mesh fixation and non-fixation in postoperative complication rates [9].

\subsection{Recurrence}

During the period of follow up (mean of 9.68 and 10.15 months in Group A and B), one case of early recurrence was discovered in Group A due to mesh displacement despite of mesh fixation with non-absorbable tacks. However, the difference between 2 groups was not statistically significant (P-value 0.335). Although biomechanical studies showed that there was significant mesh migration with mesh non-fixation [2], clinical studies revealed no increase in the risk of hernia recurrence in non-fixation group $[8,9,10]$. Moreover, studies showed no significant difference between different types of meshes or methods of mesh fixation regarding hernia recurrence $[2,11]$. 


\subsection{Pain}

Compared to traditional open techniques of inguinal herniorrhaphy, TEP has less postoperative and long-term neurologic pain $[1,12]$. Literature has some heterogeneity regarding the effect of mesh fixation on early postoperative pain. While some studies found significant reduction in early postoperative pain in mesh non-fixation $[7,10]$ and reduced need for post operative narcotic analgesia [8], a meta analysis of multiple large studies showed that postoperative pain was comparable in both groups [9]. In this study, 24 hours' postoperative pain score was significantly lower in nonfixation group (Group B; P-value 0.046). Interestingly, postoperative pain and hospital stay are significantly higher in bilateral than unilateral cases in Group A only (mesh fixation). This may be attributed to more tacks needed for mesh fixation in bilateral cases with increased possibility of nerve affection. There was positive significant correlation between postoperative pain score and operative time in both groups (Figure 9, 10).

\section{Group A}

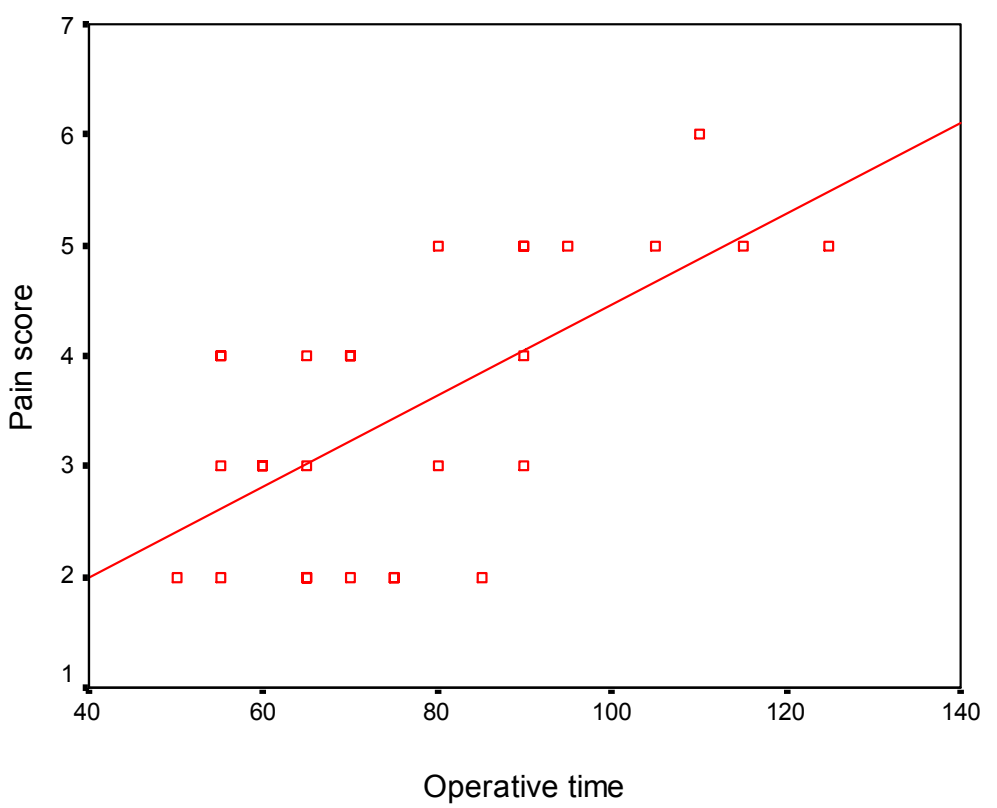

Figure 9. Positive significant correlation between Pain score and operative time in Group A.

Group B

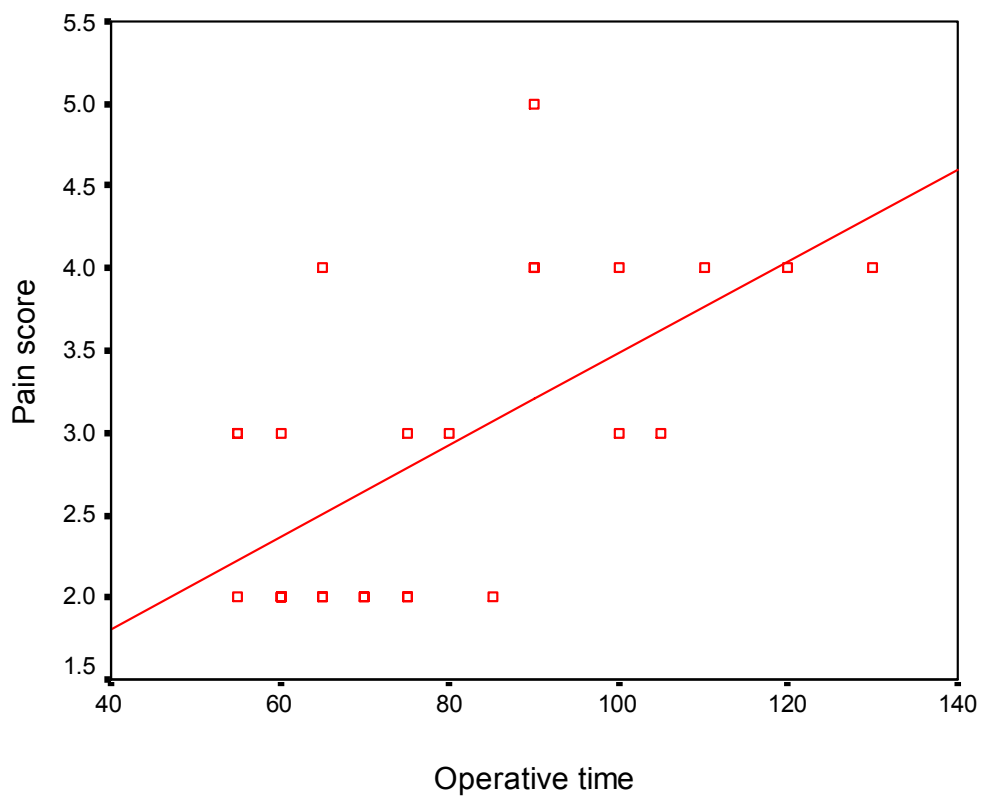

Figure 10. Positive significant correlation between Pain score and operative time in Group B. 
There was no case in this study complained from chronic groin pain in both groups during the period of follow up. Chronic pain can be defined as pain arising 3-6 months after inguinal hernia repair that can compromise the patient's quality of life [13]. The level of pain in the early postoperative period after inguinal hernia repair strongly correlates with chronic pain problems [14]. Excessive use of tacks may contribute to postoperative chronic pain [3].

\section{Conclusion}

Totally extraperitoneal repair for inguinal hernia is a safe procedure that can be a better alternative for open anterior approach repair if the expertise is available, whether with mesh fixation or non-fixation. Both options are valid, have low postoperative morbidity with comparable operative time, hospital stay and recurrence rates. Early postoperative pain is slightly higher with mesh fixation plus positive significant correlation with operative time in both groups. Larger volume studies with longer follow up periods should be done to clarify, more specifically, points like; incidence of chronic groin pain and its relation to mesh fixation, relation between drain insertion and incidence of postoperative seroma, hematoma and surgical emphysema.

\section{References}

[1] Kinga A. Powers and Daniel B. Jones (2013): Totally Extraperitoneal Inguinal Hernia Repair in: Master Techniques in Surgery, Hernia Editor: Daniel B. Jones, Josef E. Fischer, LIPPINCOTT WILLIAMS \& WILKINS 16; 173-191.

[2] Schwab R, Schwacher O, Junge K, etal., (2008): Biomechanical analysis of mesh fixation in TAPP and TEPP hernia repair, Surg. Endosc. 22 (3) 731e738.

[3] Wake BL, McCormack K, Fraser C, et al. (2005): Transabdominal pre-peritoneal (TAPP) vs totally extraperitoneal (TEP) laparoscopic techniques for inguinal hernia repair. Cochrane Database Syst Rev. (1):CD004703. DOI: $10.1002 / 14651858 . C D 004703$. pub2.

[4] Benjamin S. Powell and Guy R. Voeller (2013): Totally Extraperitoneal Inguinal Hernia Repair Using Fibrin Glue in: Master Techniques in Surgery, Hernia Editor: Daniel B. Jones,
Josef E. Fischer, LIPPINCOTT WILLIAMS \& WILKINS 17; 193-202.

[5] Osama Hasan Abd-Raboh, Taha Ahmed Ismael, Hamdy Abdel-Hady Mohamed (2017): Outcome of Laparoscopic Totally Extraperitoneal Approach with Direct Dissection and Mesh Hernioplasty in the Treatment of Inguinal Hernia Journal of Surgery 5 (3-1): 1-7.

[6] C. M. P. Claus, G. M. Rocha, A. C. L. Campos, et al. (2015): Prospective randomized and controlled study of mesh displacement after laparoscopic inguinal repair: fixation versus no fixation of mesh. Surg Endosc DOI 10.1007/s00464-015-4314-7.

[7] Mahmood Ayyaz, Muhammad Waris Farooka, Asad Ali Toor, et al. (2015): Mesh fixation vs. non-fixation in total extra peritoneal mesh hernioplasty JPMA 65: 270.

[8] Cody A. Koch, Susan M. Greenlee, Dirk R. Larson, et al. (2006): Randomized Prospective Study of Totally Extraperitoneal Inguinal Hernia Repair: Fixation Versus No Fixation of Mesh. JSLS 10:457-460.

[9] M. S. Sajid, N. Ladwa, L. Kalra, et al. (2012): A meta-analysis examining the use of tacker fixation versus no-fixation of mesh in laparoscopic inguinal hernia repair. International Journal of Surgery 10, 224e231.

[10] Ashwani Gupta, Ashish, Vimal Bhandari, et al. (2016): Comparing the Clinical Outcome of Non Fixation of Mesh with Mesh Fixation in Laparoscopic Inguinal Hernioplasty (TEP): A Study and Review of Literature Sch. J. App. Med. Sci., 4 (9D):3442-3448.

[11] Adam Cristaudo, Arun Nayak, Sarah Martin, et al. (2015): A prospective randomised trial comparing mesh types and fixation in totally extraperitoneal inguinal hernia repairs. International Journal of Surgery 17, 79e82.

[12] Tetik C, Arregui ME, Dulucq JL, et al. (1994): Complications and recurrences associated with laparoscopic repair of groin hernias. A multi-institutional retrospective analysis. Sure Endosc. 8 (11): 1316-1322.

[13] Campanelli G, Bertocchi V, Cavalli M, et al. (2013): Surgical treatment of chronic pain after inguinal hernia repair. Hernia. 17 (3):347-53.

[14] Callesen T, Bech K, Kehlet H (1999): Prospective study of chronic pain after groin hernia repair. Br J Surg 86:15281531 . 